\title{
Preface
}

\section{Recent Advances in Imaging with PET, Computed Tomography, and MR Techniques}

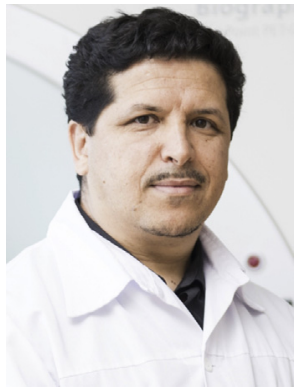

Habib Zaidi, PhD

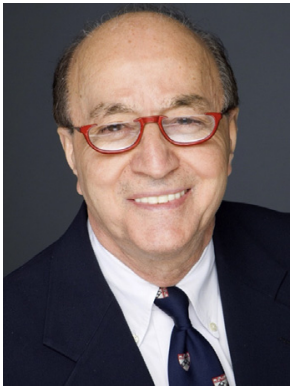

Abass Alavi, MD Editors

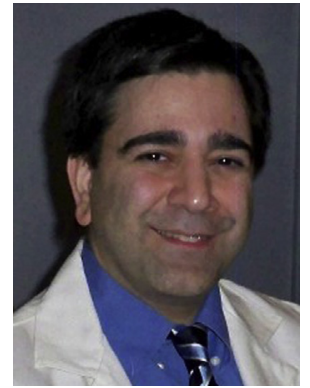

Drew A. Torigian, MD
This is an exciting time for multimodality medical imaging in the era of precision medicine. The history of medical imaging is very rich with boundless sensational developments performed by pioneers in the field. These developments cover all imaging techniques and modalities, including $x$-ray computed tomography (CT), MR imaging, and PET, and their combination on advanced hybrid imaging devices, such as PET/CT and PET/MR imaging.

Recent developments in medical imaging techniques, instrumentation, and clinical applications have created a need for a review of their role and clinical relevance in clinical and research settings. Likewise, the number of papers related to these subjects published in peer-reviewed journals and presented at various conferences and symposia has been increasing steadily, which motivated the compilation of the enclosed reviews as a snapshot of the dynamically changing field of multimodality medical imaging. This issue presents recent advances in CT, MR imaging, and PET imaging techniques, particularly total-body PET imaging instrumentation and reconstruction strategies, and summarizes state-of-the-art clinical applications of novel imaging technologies. This special issue is thus distinct from previous ones in the sense that it focuses particularly on total-body $P E T$ imaging and PET/MR imaging. Future prospects and suggestions for further research are also discussed.
The first contribution by Drs Potigailo, Kohli, Prakpoor and colleagues focuses on reviewing recent advances in anatomical CT and MR imaging modalities, including modern emerging functional (perfusion and vascular) imaging techniques. The following article by Drs Amirrashedi, Zaidi, and Ay provides a comprehensive review of preclinical PET scanners developed in academic and corporate settings with a particular emphasis on innovations in instrumentation and conceptual designs. The concept of newly introduced total-body PET imaging achieved by extending the axial field-of-view up to $2 \mathrm{~m}$, which improves substantially the sensitivity, thus allowing dose reduction, faster imaging, improved temporal resolution, and dynamic imaging, is discussed by Drs Saboury, Morris, Farhadi and colleagues. The cost of this system is the major drawback for wider clinical adoption of this technology. In this regard, the following contribution by Drs Moskal and Stępien describes J-PET, a cost-effective solution for total-body PET through the use of axially arranged low-cost plastic scintillator strips. Addressing the challenges of image reconstruction for long axial field-of-view PET scanners and dynamic imaging is systematically tackled in the contribution by Efthimiou. The clinical applications of total-body PET imaging in various fields and particularly in atherosclerosis and osteoporosis are covered in 3 comprehensive 
articles (Drs Saboury, Morris, Nikpanah and colleagues; Drs Borja, Rojulpote, Hancin and colleagues; and Drs Zhang, Koa, Borja and colleagues). The emergence of PET/MR imagingtriggered much worthwhile methodological research. This in turn spurred the exploration of the clinical potential of this novel imaging modality. As such, the clinical applications of PET/MR imagingin exploring the central nervous system (Drs Borja, Hancin, Khosravi and colleagues), cardiovascular diseases (Drs Cardoso and Leucker), and musculoskeletal diseases (Drs Hancin, Borja, Nikpanah and colleagues) are elegantly reviewed. Last, but not least, 3 articles (Drs Manhas, Salehi, Joyce and colleagues; Drs Katal, Gholamrezanezhad, Nikpanah and colleagues; Drs Nikpanah, Katal, Christensen, and colleagues) provide indepth appraisal of the role of multimodality imaging (PET/CT/MR imaging) in musculoskeletal and inflammatory diseases.

The development of advanced multimodality medical imaging instrumentation and related image reconstruction and analysis techniques as well as associated clinical applications have been very rapid and exciting, and there is every reason to believe the field will move forward more swiftly in the near future with the advent of novel crystals and photodetector technologies and the unlimited imagination of active scientists. There is no shortage of challenges and opportunities for multimodality medical imaging instrumentation and innovative clinical applications nowadays. We hope that, in this limited space, we were able to provide a flavor of recent advances in instrumentation and potential applications in clinical and research settings. We would like to thank all the authors who contributed these articles and hope that these contributions will be beneficial to readers.

Habib Zaidi, PhD

Division of Nuclear Medicine and Molecular Imaging Geneva University Hospital Geneva $\mathrm{CH}-1211$, Switzerland

Abass Alavi, MD Department of Radiology Hospital of the University of Pennsylvania

Philadelphia, PA 19104, USA

Drew A. Torigian, MD Department of Radiology University of Pennsylvania Philadelphia, PA 19104, USA

E-mail addresses:

Habib.Zaidi@hcuge.ch (H. Zaidi)

Abass.Alavi@pennmedicine.upenn.edu (A. Alavi) Drew.Torigian@pennmedicine.upenn.edu

(D.A. Torigian) 